\title{
Ségrégation de phases dans les écoulements de polymères fondus chargés en poudres métalliques
}

\author{
JeAn-Claude GÉlin ${ }^{\mathrm{a}}$ ET Thierry Barrière \\ Équipe Modélisation et Mise en Forme des Matériaux, Laboratoire de Mécanique Appliquée R. Chaléat, \\ UMR CNRS 6604/ENSMM Besançon, 24 chemin de l'épitaphe, 25030 Besançon, France
}

Reçu le 10 juin 2003, accepté le 7 novembre 2003

\begin{abstract}
Résumé - Le moulage par injection de poudres métalliques consiste à injecter des mélanges polymères/poudres métalliques. Durant l'écoulement de ces mélanges dans les moules, il peut se produire des effets de ségrégation de phases, conduisant à des défauts de formes, de propriétés physiques et mécaniques lors des étapes ultérieures du procédé. Un pilote expérimental de moulage par injection métallique, précédemment mis au point au laboratoire, est utilisé pour réaliser des composants moulés permettant d'analyser les différentes étapes du procédé. Une méthode expérimentale est développée pour déterminer la répartition de densité sur divers types de composants moulés. Une modélisation de ces phénomènes à partir de la théorie des mélanges pour les écoulements bi-phasiques est proposée. La simulation numérique des écoulements de polymères chargés en poudres solides a été développée. Enfin des exemples de simulation de remplissage, avec analyse prédictive des zones de ségrégation, sont donnés et des comparaisons calculs-expériences montrent le degré de validité de l'approche proposée.
\end{abstract}

Mots clés : Moulage / injection / polymères chargés / modèle bi-phasique / ségrégation

Abstract - Fluid and powder segregation for polymer flows with hight concentration of metallic powders. Metal injection moulding process consists to inject a mixture of a thermoplastic binder and metallic powders. During mould filling, the segregation of metallic powders and thermoplastic binder frequently arises mainly due to poor mould design. This phenomenon induces lot of defects during the subsequent stages of the process. An experimental pilot developed in the laboratory has been used to manufacture a group of moulded components. An experimental method has been developed to measure the local density for moulded components. The modelling of mould filling for metallic injection moulding is considered using a two-phase model. A numerical algorithm based on a fractional steps method has also been developed. An example of tensile test specimens filled with one or two inlets is considered. Numerical and experimental comparisons on local density values have been chosen to validate the approach.

Key words: Moulding / injection / loaded polymers / two-phases model / segregation

\section{Introduction}

Le moulage par injection de poudres (MIP) consiste à injecter des mélanges de polymères thermoplastiques chargés en poudres métalliques ou céramiques. La réalisation d'un composant par moulage par injection de poudres comprend principalement quatre étapes : le mixage d'une poudre métallique fine avec un liant thermoplastique [1], le moulage par injection qui donne la forme du composant par réplication de l'empreinte du moule [2], l'évacuation du liant ou encore déliantage qui permet

a Auteur correspondant :

jean-claude.gelin@univ-fcomte.fr d'éliminer le liant qui a joué le rôle de fluide porteur [3] et enfin la densification par diffusion à l'état solide ou par solidification/diffusion lorsqu'une partie des poudres est portée à l'état liquide [4]. La forme finale ainsi que les propriétés mécaniques des composants sont obtenues pendant l'étape de densification, mais dépendent étroitement de l'ensemble des étapes [5]. En particulier, l'écoulement de mélange fortement chargé en poudres métalliques ou céramiques fines (90\% en masse ou $60 \%$ en volume), peut provoquer durant la phase d'injection, des effets de ségrégation dont les conséquences apparaissent dans la suite du procédé $[6,7]$.

L'objectif des travaux développés est d'appréhender ces effets. Une modélisation a été développée, basée 


\section{Nomenclature}

\begin{tabular}{|c|c|c|}
\hline$X_{i}$ & variable $X$ associée à la phase $i$ du mélange & \\
\hline$\phi_{i}$ & fraction volumique de la phase $i$ & \\
\hline$\rho_{i}$ & densité courante de la phase $i$ & $\mathrm{~kg} \cdot \mathrm{m}^{-3}$ \\
\hline$\rho_{i}^{o}$ & densité initiale de la phase $i$ & $\mathrm{~kg} \cdot \mathrm{m}^{-3}$ \\
\hline $\boldsymbol{V}_{i}$ & vitesse de la phase $i$ & $\mathrm{~m} \cdot \mathrm{s}^{-1}$ \\
\hline $\boldsymbol{V}_{\text {eff }}$ & vitesse effective du mélange & $\mathrm{m} . \mathrm{s}^{-1}$ \\
\hline $\boldsymbol{V}_{m}$ & vitesse moyenne du mélange & $\mathrm{m} \cdot \mathrm{s}^{-1}$ \\
\hline $\boldsymbol{m}_{i}$ & terme d'interaction de la phase $i$ & \\
\hline$P$ & champ de pression & Mpa \\
\hline$T$ & champ de température & ${ }^{\circ} \mathrm{C}$ \\
\hline$\sigma_{i}$ & tenseur des contraintes de Cauchy de la phase $i$ & \\
\hline$\sigma_{i}^{\prime}$ & déviateur du tenseur des contraintes de Cauchy de la phase $i$ & \\
\hline$g$ & gravité & $\mathrm{m} . \mathrm{s}^{-2}$ \\
\hline$k$ & paramètre d'interaction entre phases & Pa.s.m ${ }^{-2}$ \\
\hline$\dot{\bar{\varepsilon}}_{i}$ & taux de cisaillement équivalent de la phase $i$ & \\
\hline$\dot{\bar{\varepsilon}}_{m}$ & taux de cisaillement équivalent du mélange & \\
\hline$\dot{\varepsilon}_{i}$ & tenseur des taux de déformation de la phase $i$ & \\
\hline$\dot{\varepsilon}_{i}^{\prime}$ & déviateur du tenseur des taux de déformation de la phase $i$ & \\
\hline$\mu_{i}$ & viscosité de la phase $i$ & Pa.s \\
\hline $\boldsymbol{V}_{i}^{*}$ & $\begin{array}{l}\text { vecteur prédiction de vitesse de la phase } i \text { lié à la résolution } \\
\text { de l'étape diffusion-visqueuse }\end{array}$ & \\
\hline $\boldsymbol{V}_{i}^{* *}$ & $\begin{array}{l}\text { vecteur prédiction de vitesse de la phase } i \text { lié à la résolution } \\
\text { de l'étape d'interaction }\end{array}$ & \\
\hline $\boldsymbol{V}_{i}^{n+1}$ & $\begin{array}{l}\text { vecteur prédiction de vitesse de la phase } i \text { lié à la résolution } \\
\text { de l'étape d'incompressibilité }\end{array}$ & \\
\hline$M_{i}$ & matrice masse diagonalisée de la phase $i$ & \\
\hline $\mathbf{F}_{i}$ & vecteur des forces extérieures de la phase $i$ & \\
\hline$K$ & matrice d'interaction & \\
\hline $\boldsymbol{K}_{i}$ & matrice de raideur visqueuse de la phase $i$ & \\
\hline$C$ & opérateur gradient & \\
\hline$C^{T}$ & opérateur divergence & \\
\hline$A$ & opérateur d'assemblage & \\
\hline$F(x, t)$ & variable de remplissage & \\
\hline
\end{tabular}

sur une approche bi-phasique permettant d'appréhender et de calculer la variation de fraction volumique de poudres durant l'injection $[8,9]$. Des méthodes de simulation numérique basées des algorithmes explicites à pas fractionnés ont été mises en œuvre pour développer un logiciel de simulation par éléments finis [10,11]. Un ensemble d'expérimentations a été réalisé, permettant l'obtention de composants injectés avec une très bonne reproductibilité en termes de masses et de propriétés mécaniques $[6,8]$. Des expériences ont été réalisées sur des poudres métalliques en acier inoxydable 316L avec un mélange de polymères à déliantage thermique. Un des verrous technologique en Moulage par Injection de Poudres concerne la caractérisation et la quantification de la ségrégation de phases. Seules quelques publications s'intéressent à cette caractérisation dans le domaine de la mécanique des roches [12,13]. Afin d'analyser ce problème, un équipement de mesure locale de densité a été mis en place pour évaluer précisément la distribution spatiale de fractions volumiques de poudres après injection. Les résultats concernant les distributions de fractions volumiques de poudres sont ensuite comparés aux résultats obtenus par simulation, grâce au modèle proposé et au logiciel de simulation développé.

\section{Développements expérimentaux}

\subsection{Moulage par injection}

Afin d'étudier les effets de ségrégation et de variation de répartition de poudres au cours de l'injection, un moule multi-empreintes a été conçu, instrumenté et 


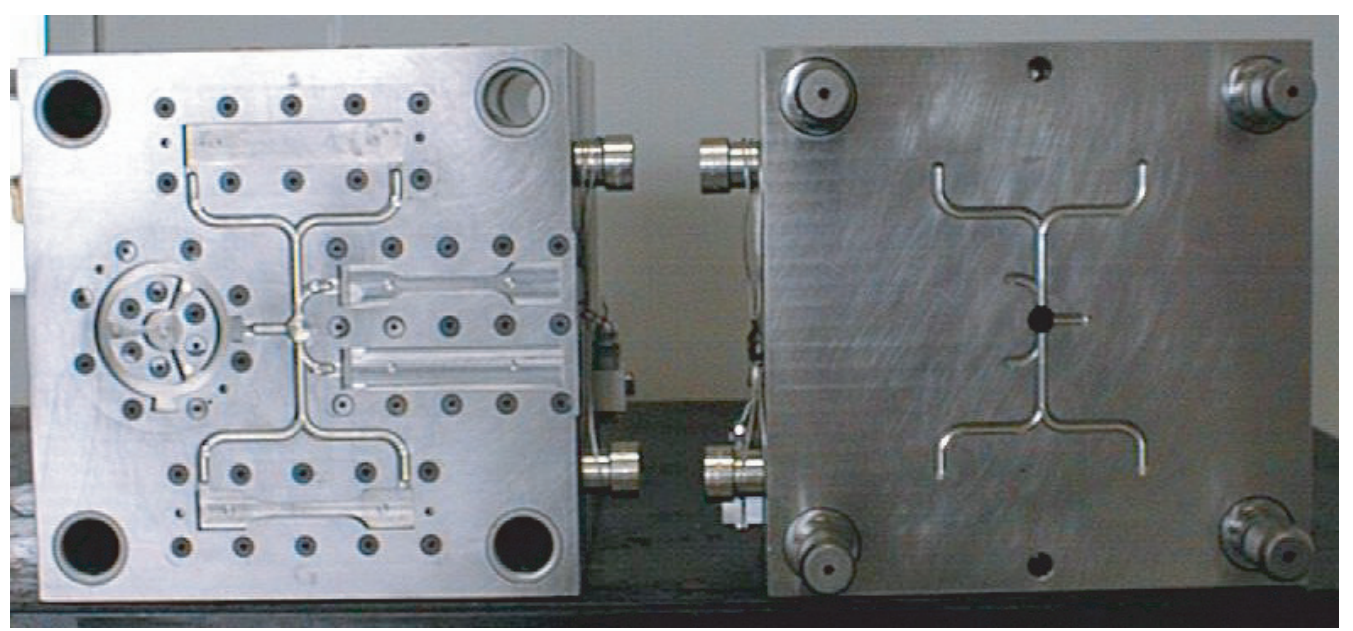

Fig. 1. Photographie des empreintes du moule.

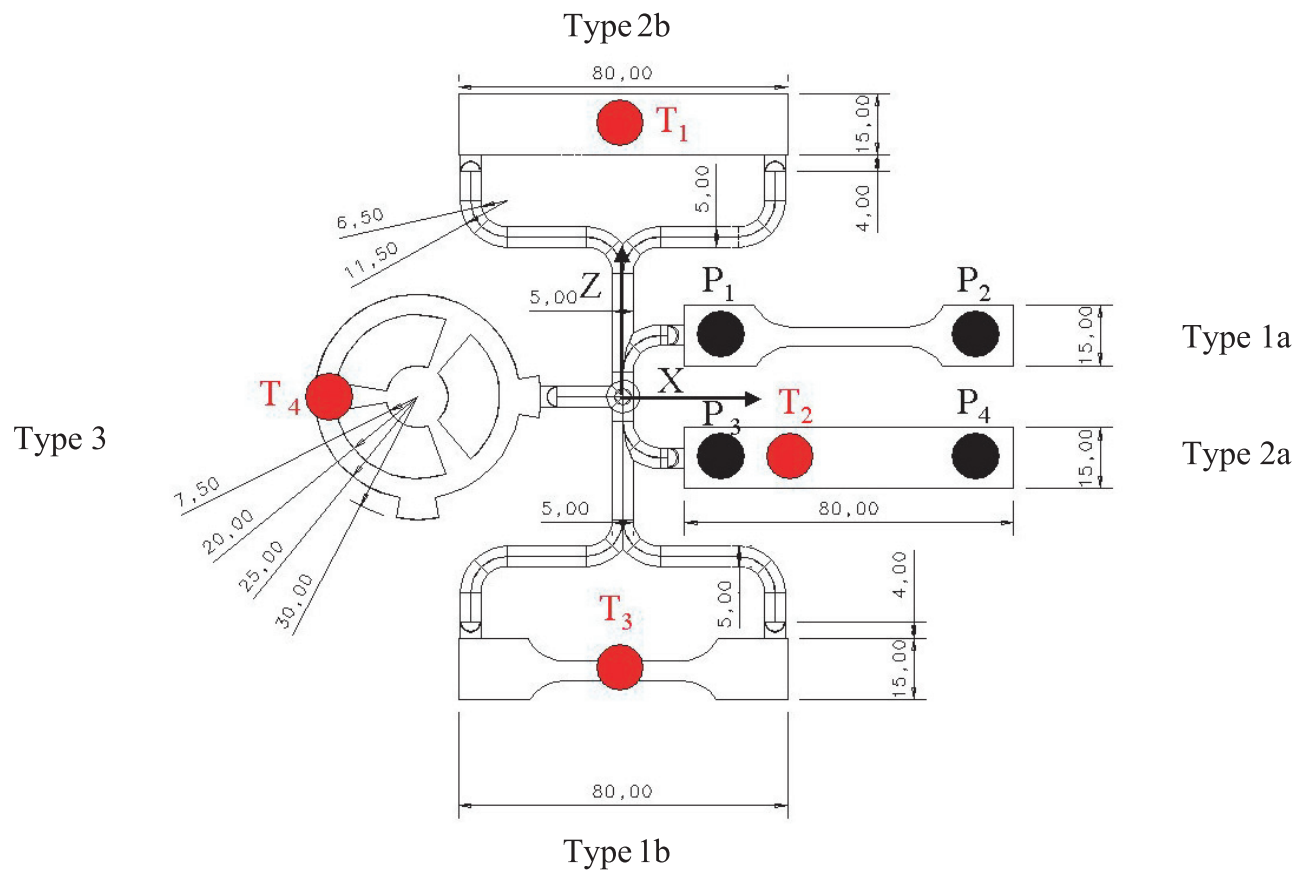

Fig. 2. Géométrie et dimensions des empreintes du moule, emplacements des capteurs de pression $\left(P_{1}\right.$ à $\left.P_{4}\right)$ et de température $\left(T_{1}\right.$ à $\left.T_{4}\right)$.

réalisé au laboratoire. Il permet de réaliser des composants destinés à différents usages : éprouvettes destinées à la mesure des propriétés mécaniques, éprouvettes conçues pour étudier le déliantage et la densification, ou encore empreintes pour tests d'injectabilité [4].

La description de la conception et la réalisation du moule est donnée dans $[6,7]$. Le moule réalisé comprend 5 empreintes permettant de réaliser 3 types de composants : des éprouvettes de traction avec un ou deux points d'injection (type 1a et 1b), des éprouvettes de flexion avec un ou deux points d'injection (type $2 \mathrm{a}$ et $2 \mathrm{~b}$ ) et un composant de type volant (type 3 ) avec plusieurs zones de jonction de fronts d'écoulements, figures 1 et 2 .

Les composants ainsi réalisés (comprenant un ou deux points d'injection pour un même composant) permettent de quantifier les effets des paramètres d'injection ainsi que la position des zones de ségrégations éventuelles durant la phase d'injection par rapport aux points d'injection.

L'alimentation des empreintes en mélange de polymères chargés en poudres est très importante pour le bon déroulement de l'injection, ainsi le dimensionnement des canaux et seuils a été réalisé à partir de simulations numériques par éléments finis, en utilisant une viscosité équivalente pour le mélange [14]. Les résultats de simulation conduisent à des canaux de section circulaire de $5 \mathrm{~mm}$ de diamètre et à des seuils de $6 \mathrm{~mm}^{2}$ de section.

Le moulage des composants est réalisé avec une presse à injecter de type $\mathrm{BOY}^{\circledR} 22 \mathrm{~T}$. Les paramètres d'injection correspondent à une pression d'injection $16 \mathrm{MPa}$ et à une vitesse d'injection de $160 \mathrm{~mm} . \mathrm{s}^{-1}$. La machine est 


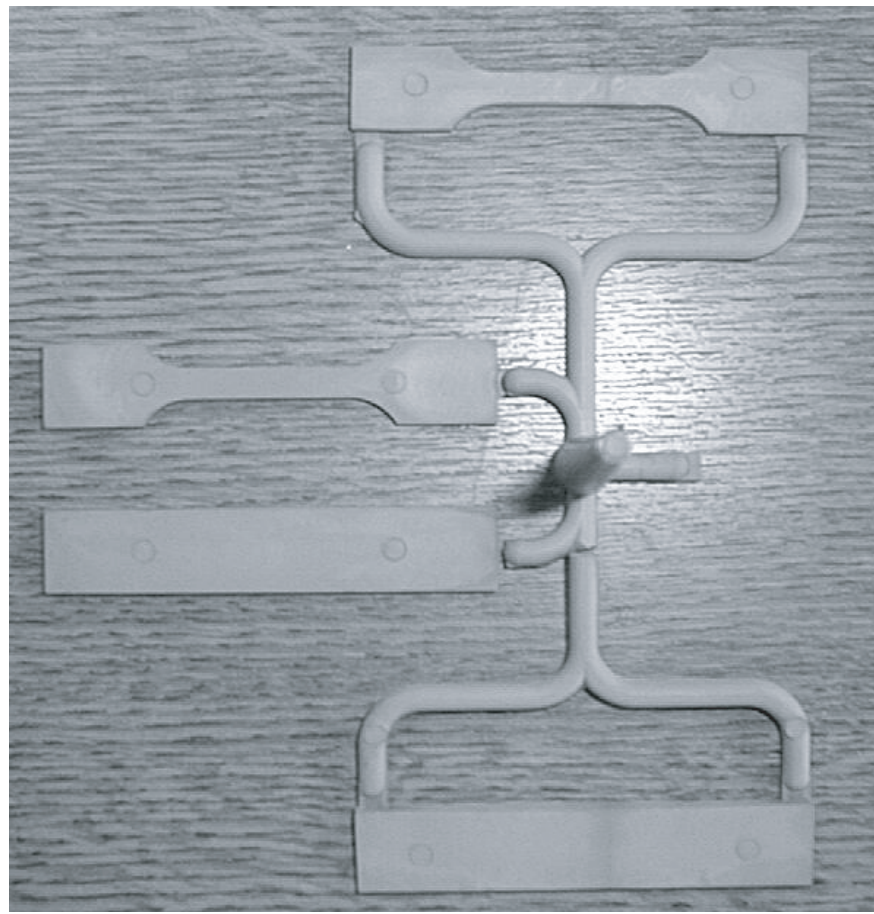

Fig. 3. Photographie des composants obtenus grâce au moule multi-empreintes. À partir de mélange à base de poudres métalliques $316 \mathrm{~L}$.

Tableau 1. Paramètres d'injection utilisés pour le mélange à base de poudres métalliques $316 \mathrm{~L}$.

\begin{tabular}{lcc}
\hline & $\begin{array}{c}\text { Paramètres } \\
\text { utilisés }\end{array}$ & {$[15]$} \\
\hline Température d'injection du mélange & $175{ }^{\circ} \mathrm{C}$ & $175^{\circ} \mathrm{C}$ \\
Température du moule & $48{ }^{\circ} \mathrm{C}$ & $45^{\circ} \mathrm{C}$ \\
Temps d'injection & $1 \mathrm{~s}$ & $\leq 1 \mathrm{~s}$ \\
Vitesse d'injection & $160 \mathrm{~mm} \cdot \mathrm{s}^{-1}$ & \\
Pression d'injection & $16 \mathrm{MPa}$ & \\
Pression de maintien & $6 \mathrm{MPa}$ & $6,5 \mathrm{MPa}$ \\
Temps de refroidissement & $20 \mathrm{~s}$ & \\
\hline
\end{tabular}

équipée d'une vis de diamètre de $22 \mathrm{~mm}$ avec une course maximale d'injection de $80 \mathrm{~mm}$. La force maximale de fermeture est de $22000 \mathrm{~N}$. Elle possède quatre zones de chauffage pour fondre le polymère. Les paramètres d'injection utilisés sont donnés tableau 1. Les composants obtenus sont donnés figure 3. Les paramètres de moulage correspondent à ceux proposés par le fournisseur du matériau [15] et correspondent également à des travaux réalisés précédemment au sein du laboratoire. Des thermocouples et des capteurs de pression placés dans les empreintes du moule permettent de s'assurer des conditions et paramètres d'injection (Fig. 2). Le temps d'injection est comparable à celui obtenu par d'autres auteurs pour réaliser le même type de composants [15-18].

L'évolution de la température du mélange pendant un cycle complet de moulage est représentée figure 4. La température maximale dans l'éprouvette de type 2a est de $165^{\circ} \mathrm{C}$ (capteur $T_{2}$ ), pour une consigne de température de $175 \pm 10^{\circ} \mathrm{C}$. La température maximale dans l'éprouvette de type $2 \mathrm{~b}$ est de $131{ }^{\circ} \mathrm{C}$ (capteur $T_{1}$ ). La pression maximale dans l'éprouvette de type 2a est de 19,35 MPa (capteur $\left.P_{3}\right)$. La répétitivité des valeurs expérimentales maximales a été évaluée afin de quantifier la dispersion des mesures. Les résultats sont indiqués figure 5. On observe une bonne répétitivité des mesures de température et de pression pendant l'injection pour le mélange à déliantage thermique utilisé.

Les composants obtenus possèdent des formes correctes, et sont exempts de défauts extérieurs, voir figure 3.

\subsection{Mesures de densité}

Les mesures de densité sont réalisées à l'aide d'un pycnomètre hélium. Afin d'obtenir les résultats les plus précis possibles, plusieurs mesures sont effectuées pour obtenir une valeur moyenne de densité, le temps de réalisation de ces mesures est d'environ 30 minutes. La densité du mélange composé de poudres en acier inoxydable 316L et des différents liants, avant moulage, est de $5,16 \mathrm{~g} \cdot \mathrm{cm}^{-3}$.

Une éprouvette de traction de chaque type (type 1a avec un seul point d'injection ou type $1 \mathrm{~b}$ avec deux points d'injection) est découpée en plusieurs segments afin d'effectuer des mesures locales de densité, figure 6 .

Les résultats des mesures de densité sont représentés figure 7 . Une densité relativement homogène de poudre est obtenue, on remarque néanmoins que des valeurs plus importantes sont obtenues au niveau des seuils. Une valeur maximale de 5,30 est obtenue dans le cas d'une cavité avec un point d'injection, par rapport à 5,33 dans le cas d'une cavité avec deux points d'injection.

\section{Modélisation et simulation numérique de la ségrégation de phases}

Il n'existe pas actuellement de logiciels commerciaux capables de simuler le remplissage de moule en Moulage par Injection de Poudres Métalliques, et en particulier pas de logiciels capables de simuler la ségrégation de phases.

Bien que différentes approches physiques puissent être appliquées, la théorie des écoulements des matériaux granulaires n'est évidemment pas utilisable pour des simulations 3D de l'étape d'injection, du fait qu'elle considère l'évolution de chacune des particules dans le fluide [19], ce qui conduit à des temps de calcul très importants. Les modélisations et simulations réalisées en considérant le mélange polymère + poudres comme un fluide monophasique en introduisant une viscosité équivalente [20], ne peuvent pas représenter la ségrégation de phases de façon précise, du fait qu'elles ne contiennent pas les équations nécessaires pour rendre compte des variations de fraction volumique.

Plusieurs auteurs $[6,8,12,14,20-22]$ ont utilisé le cadre de la théorie des mélanges et ont développé une approche d'écoulements bi-phasiques associée, pour rendre compte de la variation de fraction volumique de poudre durant et en fin d'injection. Cette approche considère 


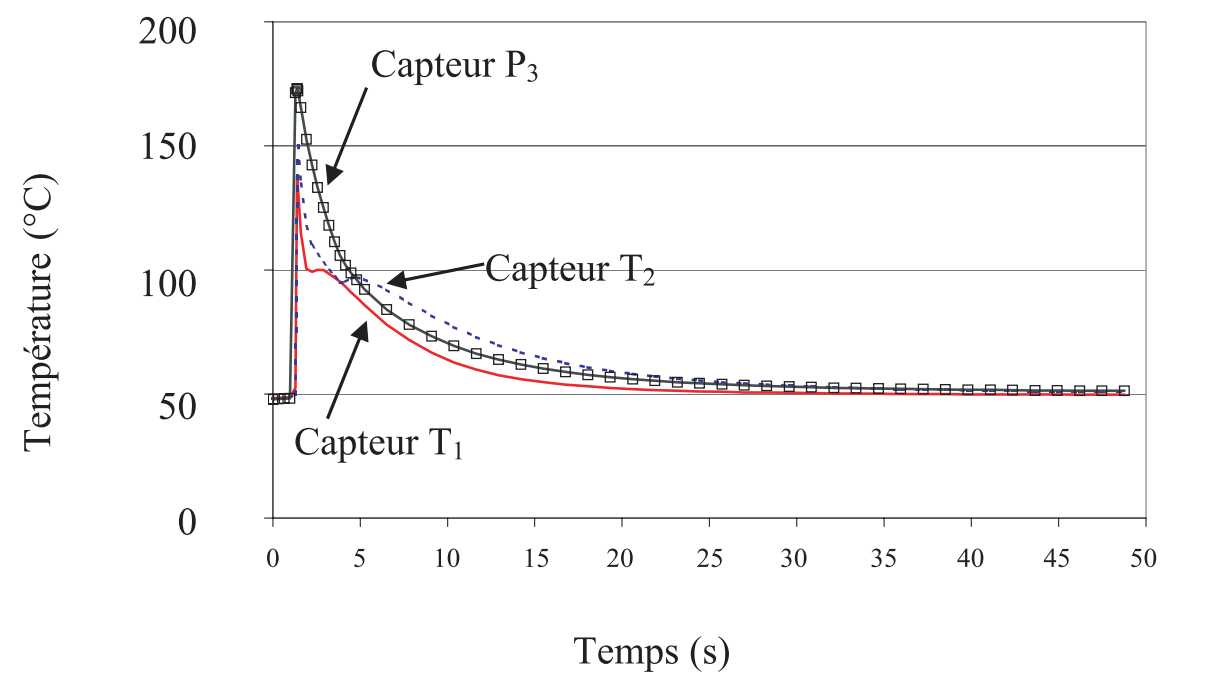

Fig. 4. Variation de la température et de la pression lors de l'injection du mélange $316 \mathrm{~L}$ à déliantage thermique $\left(P_{i n j}=16 \mathrm{MPa}\right.$, $T_{\text {inj }}=175{ }^{\circ} \mathrm{C}, T_{\text {paroi }}=48{ }^{\circ} \mathrm{C}$.

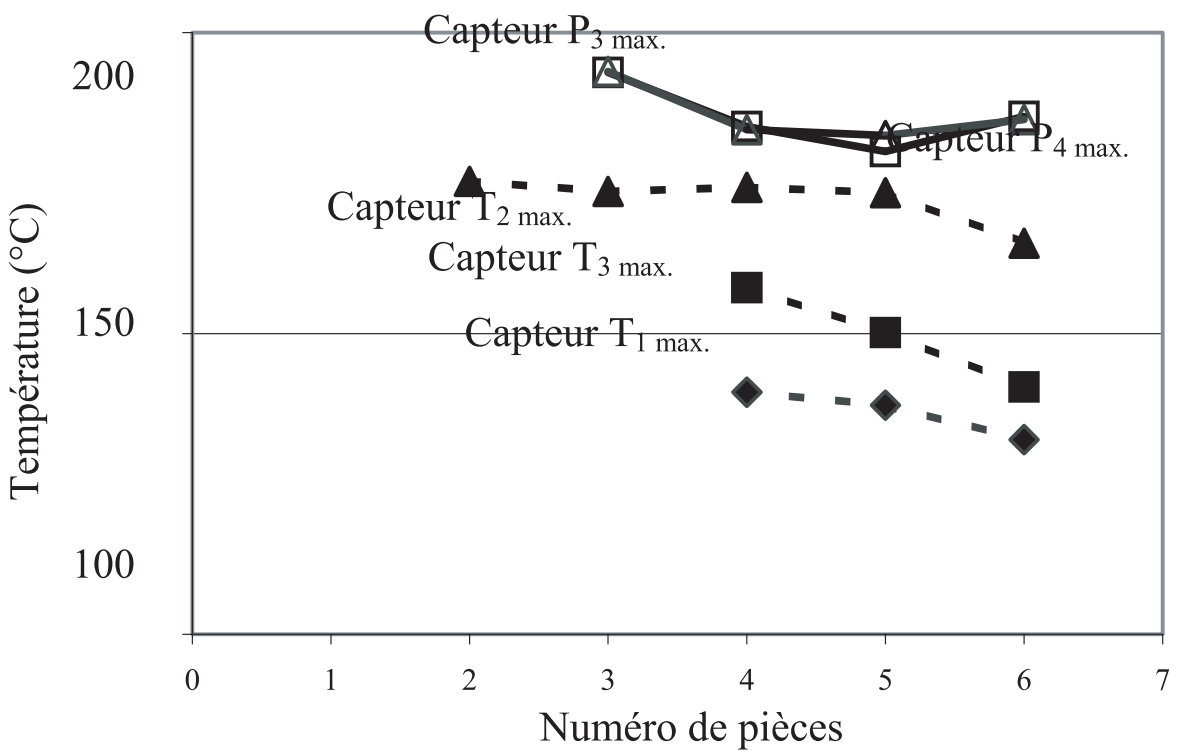

Fig. 5. Évolution des pressions et températures pour différents échantillons (316L à déliantage thermique, $P_{i n j}=16 \mathrm{MPa}$, $\left.T_{\text {inj }}=175^{\circ} \mathrm{C}, T_{\text {paroi }}=48{ }^{\circ} \mathrm{C}\right)$.

l'écoulement de deux phases distinctes : une phase dite solide considérée comme un fluide visqueux représentant les poudres, et une phase fluide représentant le polymère fondu. L'interaction entre les deux phases et l'incompressibilité du mélange sont prises en compte dans la représentation de l'écoulement du mélange poudre + liant. Cette théorie est bien adaptée à la simulation de l'écoulement des fluides visqueux fortement chargés en poudres [21,22], elle est par conséquent bien adaptée au cas du MIM. Chacune des phases possède sa propre loi de comportement et les équations de conservation sont écrites pour chacune des phases. L'interaction entre les deux phases est représentée par un coefficient d'interaction [11,21,22].

Plusieurs auteurs ont utilisé des algorithmes implicites pour résoudre les problèmes d'écoulements bi-phasiques $[21,22]$ en remplissage de moules. Les auteurs considèrent le problème comme un problème transitoire dynamique et le résolve de manière itérative et incrémentale. Le système algébrique couplé, associé au modèle bi-phasique est transitoire, non linéaire et de grande taille. Il y a donc des risques de non convergence; de plus, le temps de calcul est très important. C'est la raison pour laquelle nos développements se sont orientés vers la mise en ouvre et l'utilisation d'un modèle dynamique transitoire explicite, adapté à partir du modèle développé pour le moulage par injection de polymères fondus.

Considérant le cas de la simulation de remplissage de moules en fonderie, certains auteurs ont proposé d'utiliser des algorithmes dynamiques explicites, développés en particulier pour des simulations tri-dimensionnelles [23-25]. Les mêmes auteurs ont proposé d'utiliser des méthodes 


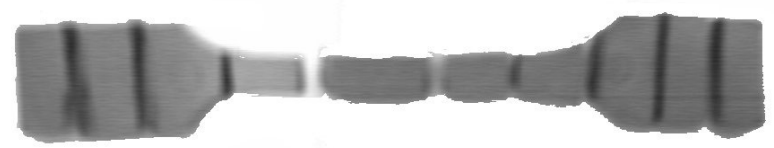

Entrée

(a) Eprouvette type 1a

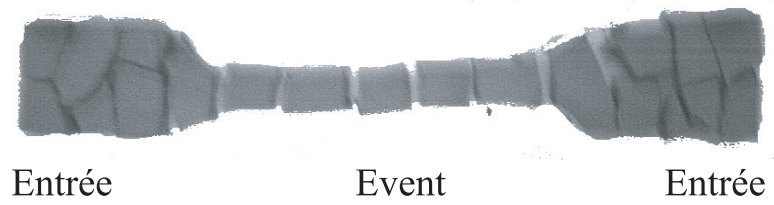

(b) Eprouvette type 1b

Fig. 6. Segmentation des éprouvettes de traction, (a) éprouvette injectée avec un point d'injection, (b) éprouvette injectée avec deux points d'injection (pour le mélange liant + poudre $316 \mathrm{~L})$.

explicites pour calculer de manière découplée l'ensemble des variables vitesse, pression et température ainsi que l'état de remplissage. Le calcul du champ de pression est effectué par une méthode globale sans itération, aboutissant à un système avec un nombre de degrés de libertés moins important que celui du champ de vitesse $[25,26]$.

\section{1 Équations de conservation}

\subsubsection{Conservation de la masse}

L'écoulement du mélange est représenté à l'aide d'une phase solide pour la poudre tandis que le liant est représenté par une phase fluide. Les deux phases sont caractérisées respectivement par les fractions volumiques solide et fluide $\left(\phi_{s}, \phi_{f}\right)$, devant respecter la condition de saturation pour le mélange $\phi_{s}+\phi_{f}=1$. La variation fraction volumique de chaque phase permet de représenter l'évolution de la ségrégation au cours du remplissage. Les densités de chacune des phases sont définies par les relations $\rho_{s}=\phi_{s} \rho_{s}^{o}$ et $\rho_{f}=\phi_{f} \rho_{f}^{o}$ où $\rho_{s}^{o}, \rho_{f}^{o}$ caractérisent les densités des phases solide et fluide denses supposées constantes. En utilisant ces définitions, la conservation de la masse pour chaque phase s'écrit sous la forme :

$$
\frac{\partial \phi_{s}}{\partial t}+\nabla \bullet\left(\phi_{s} \boldsymbol{V}_{s}\right)=0 \quad \text { et } \quad \frac{\partial \phi_{f}}{\partial t}+\nabla \bullet\left(\phi_{f} \boldsymbol{V}_{f}\right)=0
$$

où $\boldsymbol{V}_{s}$ et $\boldsymbol{V}_{f}$ représentent respectivement les vitesses de la phase solide et fluide.

En utilisant la condition de saturation dans chacune des équations précédentes, la condition d'incompressibilité du mélange s'écrit sous la forme :

$$
\nabla \bullet \boldsymbol{V}_{\text {eff }}=0 \quad \text { avec } \quad \boldsymbol{V}_{\text {eff }}=\phi_{s} \boldsymbol{V}_{s}+\phi_{f} \boldsymbol{V}_{f}
$$

où $\boldsymbol{V}_{\text {eff }}$ est la vitesse effective du mélange.

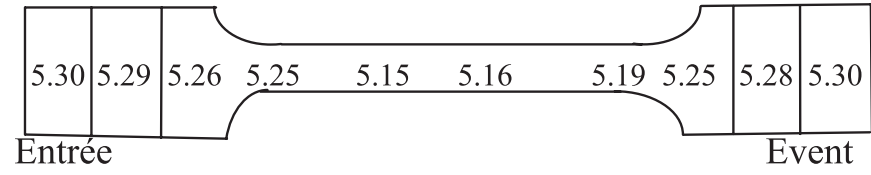

(a) Eprouvette type 1a

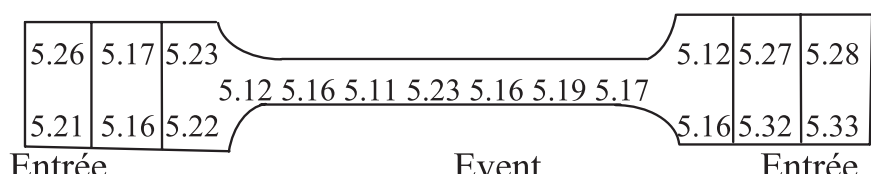

(b) Eprouvette type 1b

Fig. 7. Mesures expérimentales de la densité dans les éprouvettes de traction, (a) éprouvette injectée avec un point d'injection, (b) éprouvette injectée avec deux points d'injection (pour le mélange liant + poudre 316L) (densité en g.cm $\left.{ }^{-3}\right)$.

\subsubsection{Conservation de la quantité de mouvement}

En considérant que dans le cas du MIM les termes d'advection puissent être négligés [11, 14,21,22], les équations de conservation de la quantité de mouvement des phases solide et fluide s'écrivent séparément à l'aide de deux équations de Stokes avec des termes d'interaction opposés $\boldsymbol{m}_{s}=-\boldsymbol{m}_{f}$ :

$$
\begin{aligned}
\rho_{s} \frac{\partial \boldsymbol{V}_{s}}{\partial t}=-\nabla\left(\phi_{s} P\right)+\nabla \bullet \boldsymbol{\sigma}_{s}^{\prime}+\rho_{s} \boldsymbol{g}+\boldsymbol{m}_{s} \\
\text { et } \quad \rho_{f} \frac{\partial \boldsymbol{V}_{f}}{\partial t}=-\nabla\left(\phi_{f} P\right)+\nabla \bullet \boldsymbol{\sigma}_{f}^{\prime}+\rho_{f} \boldsymbol{g}+\boldsymbol{m}_{f}
\end{aligned}
$$

où $P$ représente le champ de pression, $\boldsymbol{\sigma}_{s}^{\prime}$ et $\boldsymbol{\sigma}_{f}^{\prime}$ sont les déviateurs des tenseurs des contraintes partielles de Cauchy des phases solide et fluide et $\boldsymbol{g}$ la gravité. Les termes d'interaction s'écrivent sous la forme :

$$
\boldsymbol{m}_{s}=k\left(\boldsymbol{V}_{f}-\boldsymbol{V}_{s}\right) \quad \text { et } \quad \boldsymbol{m}_{f}=k\left(\boldsymbol{V}_{s}-\boldsymbol{V}_{f}\right)
$$

où $k$ est un paramètre d'interaction entre les phases.

\subsection{3 Équation thermique}

L'équation de couplage thermique s'écrit sous la forme :

$$
\rho_{m} c_{m}\left(\frac{\partial T_{m}}{\partial t}+\boldsymbol{V}_{m} \bullet \nabla T_{m}\right)=k \Delta T_{m}+s_{m}
$$

Les coefficients et variables homogénéisés du mélange peuvent être exprimés sous la forme :

$$
\begin{gathered}
\rho_{m} c_{m}=\phi_{f} \rho_{f} c_{f}+\phi_{S} \rho_{S} c_{S} \\
\rho_{m} c_{m} V_{m}=\phi_{f} \rho_{f} c_{f} V_{f}+\phi_{S} \rho_{S} c_{S} V_{p} \\
k_{m}=\phi_{f} k_{f}+\phi_{S} k_{S}
\end{gathered}
$$


où $\rho_{m}$ est la densité du mélange, $\rho_{i}$ la densité de la phase $i$, $c_{m}$ la capacité calorifique du mélange, $c_{i}$ la capacité calorifique de la phase $i, k_{m}$ la conductivité thermique du mélange, $\rho_{i}$ la conductivité thermique de la phase $i$.

Dans ces relations, la vitesse de transport $V_{m}$ a une expression qui est notablement différente de la vitesse volumique définie précédemment et de la vitesse massique du mélange $\left(\rho_{m} V_{m}=\phi_{f} \rho_{f} V_{f}+\phi_{S} \rho_{S} V_{p}\right)$ [19]. L'expression des autres paramètres est donnée par une simple loi des mélanges.

\subsubsection{Lois de comportement}

Les lois de comportement visqueuses représentant le comportement de chaque phase sont écrites sous la forme de lois visqueuses :

$$
\boldsymbol{\sigma}_{s}^{\prime}=2 \mu_{s}\left(\phi_{s}, T, \dot{\bar{\varepsilon}}_{s}^{\prime}\right) \dot{\varepsilon}_{s}^{\prime} \quad \text { et } \quad \boldsymbol{\sigma}_{f}^{\prime}=2 \mu_{f}\left(\phi_{f}, T, \dot{\varepsilon}_{f}^{\prime}\right) \dot{\varepsilon}_{f}^{\prime}
$$

où $\dot{\varepsilon}_{s}^{\prime}$ et $\dot{\varepsilon}_{f}^{\prime}$ sont respectivement les déviateurs des tenseurs des taux de déformation de la phase solide et fluide, ces deux grandeurs sont calculées à partir du champ de vitesse pour chaque phase, $\mu_{s}$ et $\mu_{f}$ représentent les viscosités respectives de chacune des phases, $T$ la température du mélange, le taux de cisaillement équivalent de la phase $i$ étant défini par $\dot{\bar{\varepsilon}}_{i}=\sqrt{2 / 3}\left(\dot{\varepsilon}_{i}^{\prime}: \dot{\varepsilon}_{i}^{\prime}\right)^{1 / 2}$.

\subsection{Simulation numérique}

Les équations de Stokes sont discrétisées par la méthode des éléments finis. La résolution de ces équations se fait de manière explicite par une nouvelle méthode proposée et développée pour le traitement de l'incompressibilité du mélange $[6,9,10]$.

\subsubsection{Diffusion visqueuse}

Après discrétisation par la méthode de Galerkin, l'effet de la diffusion visqueuse est pris en compte dans la première étape de l'algorithme de résolution à pas fractionnés afin de déterminer une première prédiction des champs de vitesse $\boldsymbol{V}_{S}^{*}$ et $\boldsymbol{V}_{f}^{*}$ :

$$
\begin{aligned}
\boldsymbol{M}_{S} \frac{\boldsymbol{V}_{S}^{*}-\boldsymbol{V}_{S}^{n}}{\Delta t}= & \boldsymbol{K}_{S} \boldsymbol{V}_{S}^{n}+\boldsymbol{F}_{S} \\
& \text { et } \quad \boldsymbol{M}_{f} \frac{\boldsymbol{V}_{f}^{*}-\boldsymbol{V}_{f}^{n}}{\Delta t}=\boldsymbol{K}_{f} \boldsymbol{V}_{f}^{n}+\boldsymbol{F}_{f}
\end{aligned}
$$

où $\boldsymbol{M}_{S}$ et $\boldsymbol{M}_{f}$ sont les matrices masses diagonalisées de chacune des phases, $\boldsymbol{K}_{S}$ et $\boldsymbol{K}_{f}$ sont les matrices de raideur visqueuse, $\boldsymbol{F}_{S}$ et $\boldsymbol{F}_{f}$ sont les vecteurs des forces extérieures.

\subsubsection{Effets d'interaction}

La seconde étape de l'algorithme à pas fractionnés consiste à prendre en compte les effets d'interaction entre phases. À l'aide d'une matrice d'interaction $\boldsymbol{K}$, la discrétisation conduit à deux équations pour une nouvelle prédiction des champs de vitesse $\boldsymbol{V}_{S}^{* *}$ et $\mathbf{V}_{f}^{* *}$ :

$$
\begin{aligned}
& \boldsymbol{M}_{S} \frac{\boldsymbol{V}_{S}^{* *}-\boldsymbol{V}_{S}^{*}}{\Delta t}=\boldsymbol{K}\left(\boldsymbol{V}_{f}^{n}-\boldsymbol{V}_{S}^{n}\right) \\
& \text { et } \quad \boldsymbol{M}_{f} \frac{\boldsymbol{V}_{f}^{* *}-\boldsymbol{V}_{f}^{*}}{\Delta t}=\boldsymbol{K}\left(\boldsymbol{V}_{S}^{n}-\boldsymbol{V}_{f}^{n}\right)
\end{aligned}
$$

\subsection{Champ de pression et incompressibilité du mélange}

Le traitement de l'incompressibilité se concrétise dans l'algorithme à pas fractionnés par un effet du champ de pression sur les champs de vitesse. Après discrétisation, on obtient respectivement les équations suivantes pour chacune des phases :

$$
\begin{aligned}
& \boldsymbol{M}_{S} \frac{\boldsymbol{V}_{S}^{n+1}-\boldsymbol{V}_{S}^{* *}}{\Delta t}=-\phi_{S} \boldsymbol{C P} \\
& \text { et } \quad \boldsymbol{M}_{f} \frac{\boldsymbol{V}_{f}^{n+1}-\boldsymbol{V}_{f}^{* *}}{\Delta t}=-\phi_{f} \boldsymbol{C P}
\end{aligned}
$$

Par addition des équations (8) et en tenant compte de l'incompressibilité du mélange (2), le champ de pression $P$ est déterminé grâce à une résolution globale, sous la forme :

$$
A C^{T} M_{e q}^{-1} C P=\frac{1}{\Delta t} A C^{T} \boldsymbol{V}_{e f f}^{* *}
$$

où $C$ est l'opérateur gradient, tandis que sa transposée $C^{T}$ représente l'opérateur divergence, $A$ est l'opérateur d'assemblage sur l'ensemble des éléments finis du domaine considéré. Les champs de vitesse $\boldsymbol{V}_{S}^{n+1}$ et $\boldsymbol{V}_{f}^{n+1}$ sont facilement obtenus grâce à l'équation (10) et à la connaissance du champ de pression.

\subsection{Détermination des fractions volumiques de poudre et de liant}

En tenant compte de la condition de saturation, les fractions volumiques se calculent grâce aux équations (1a, b), ces équations sont discrétisées par une méthode de Galerkin [6,9].

\subsection{Calcul de la position du front}

Dans le cadre d'une description eulérienne, un champ $F(x, t)$ est utilisé pour décrire l'état de remplissage. Cette variable prend une valeur 1 dans le domaine rempli et 
0 dans le domaine vide, $F$ est déterminé par une équation de diffusion advection de la forme :

$$
\frac{\partial F}{\partial t}+\nabla \bullet\left(\boldsymbol{V}_{\text {eff }} F\right)=0
$$

La méthode de Taylor-Galerkin est utilisée pour résoudre cette équation afin d'éviter d'éventuelles oscillations numériques dues aux effets de convection [26].

\section{Détermination des paramètres rhéologiques}

L'utilisation du modèle bi-phasique pour la simulation par éléments finis nécessite une détermination des paramètres rhéologiques pour l'écoulement de chacune des phases, ainsi que leur interaction. Mais seule la viscosité du mélange (polymère fondu + poudres métalliques) peut être obtenue par des essais de rhéométrie capillaire ou grâce à une buse de rhéologie permettant de réaliser des essais in situ sur la machine d'injection. Des recherches antérieures ont montré que la viscosité de chacune des phases d'un modèle bi-phasique est loin d'être la viscosité de chaque constituant. À partir de la conservation de masse, l'écoulement obtenu par le modèle bi-phasique est équivalent à l'écoulement réel du mélange. Il implique que la détermination de la viscosité de chacune des phases dans le cadre mathématique du modèle bi-phasique conduise à un écoulement équivalent à l'écoulement du mélange réellement observé. Des recherches antérieures supposent que la viscosité du fluide est nulle, mais cette hypothèse ne s'adapte pas au cas de l'injection métallique avec un liant polymère. La détermination des paramètres visqueux pour le modèle biphasique se réalise en trois étapes successives : la première étape consiste en la mesure de la viscosité du mélange pour différentes températures et taux de cisaillements grâce à un viscosimètre capillaire, elle permet l'identification de la viscosité du mélange, la deuxième étape consiste en l'analyse d'un écoulement de Poiseuille plan, à partir d'un modèle analytique bi-phasique, pour déterminer la viscosité de chacune des phases dans les mêmes conditions de température et de taux de cisaillement. Cette analyse est basée sur le principe que la conservation de masse soit vérifiée tant par l'écoulement bi-phasique que par l'écoulement du mélange, cette condition doit être respectée pour différentes tailles de capillaire. La troisième étape consiste à déterminer le paramètre d'interaction en utilisant les lois de viscosités déterminées pour chacune des phases. Un essai de ségrégation pouvant être utilisé pour identifier le coefficient d'interaction par couplage calculs-expériences à l'aide d'une méthode inverse.

\section{Étape 1 : identification de la viscosité du mélange}

Les essais sont effectués pour différents taux de cisaillement et à différentes températures. La viscosité du mélange est fonction de la température $T$, de la fraction volumique initiale de poudre $\Phi_{s}$ et du taux de cisaillement

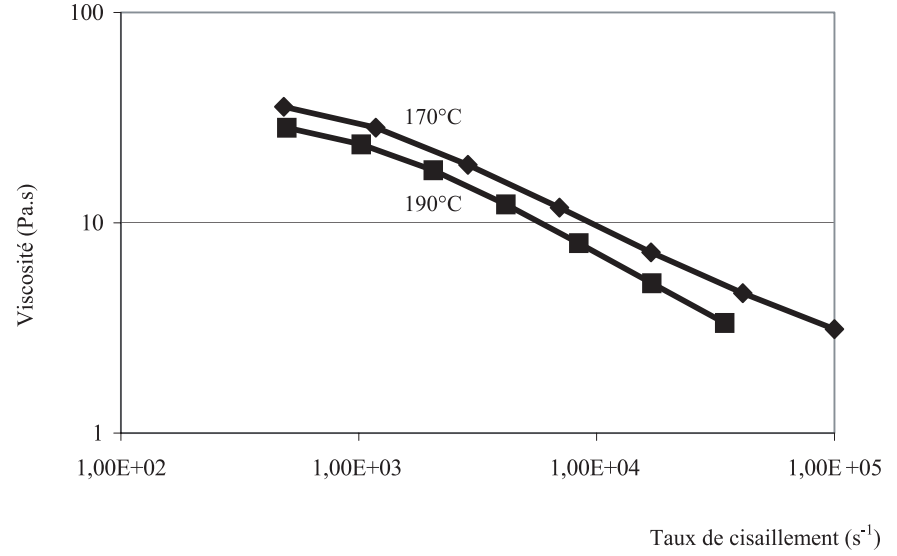

Fig. 8. Viscosité de mélange $316 \mathrm{~L}$ à déliantage thermique pour deux températures distinctes.

équivalent $\dot{\bar{\varepsilon}}_{m}$. La relation de comportement en résultant s'écrit symboliquement sous la forme suivante :

$$
\boldsymbol{\sigma}_{m}^{\prime}=2 \mu_{m}\left(T, \phi_{s}, \dot{\bar{\varepsilon}}_{m}\right) \dot{\varepsilon}_{m}^{\prime}
$$

Deux essais de rhéologie capillaire ont été réalisés pour un mélange $316 \mathrm{~L}$ à déliantage thermique pour deux températures correspondant à $170{ }^{\circ} \mathrm{C}$ et $190^{\circ} \mathrm{C}$. L'évolution de la viscosité du mélange en fonction du taux de cisaillement, pour les deux températures considérées, est relatée figure 8 .

\section{Étape 2 : détermination de la viscosité de chacune des phases}

Pour obtenir un modèle relativement simple permettant l'identification de la viscosité de chacune des phases, on considère l'écoulement de Poiseuille plan dans le cadre de la théorie des mélanges, par souci de simplicité. Un modèle analytique quasi-statique de Poiseuille plan est donc utilisé pour déterminer la correspondance entre l'écoulement du mélange et l'écoulement biphasique $[21,22]$. Une condition de paroi collante est utilisée pour l'écoulement de chacune des phases afin de se placer dans des hypothèses similaires à celles utilisées dans les essais de rhéométrie capillaire, figure 9. La solution analytique de ce modèle est donnée tableau 2. Dans les mêmes conditions de température et taux de cisaillement en parois, la conservation de masse doit être vérifiée par la relation $\boldsymbol{V}_{m}=\phi_{s} \boldsymbol{V}_{s}+\phi_{f} \boldsymbol{V}_{f}$ dans laquelle $\boldsymbol{V}_{m}$ représente la vitesse du mélange. Cette relation doit être respectée pour différents diamètres de capillaire, pour un même état rhéologique (température et taux de cisaillement en parois). Evidemment différentes valeurs de pressions doivent être appliquées pour les différents capillaires en vue de conserver le même taux de cisaillement en parois. L'analyse montre bien que le choix du paramètre d'interaction est indépendant du choix de viscosité de chacune des phases, si toutefois l'effet de ségrégation est supposé rester faible. Sous cette condition, le choix de la viscosité de chacune des phases doit conduire à la vérification de la conservation de masse entre l'écoulement bi-phasique (2) et l'écoulement observé du mélange. 
Tableau 2. Méthode d'identification à partir de l'écoulement de type Poiseuille.

Résolution du problème : écoulement de Poiseuille en capillaire plan

$\mathrm{y}=\mathrm{h}, \quad \dot{\gamma}_{\mathrm{xy}}=\frac{\Delta \mathrm{P} \mathrm{h}}{2 \mu_{\mathrm{m}} \mathrm{L}}$

Hypothèse :

Le mélange, la phase solide et fluide sont collants à la paroi

\section{Champs de vitesses solution :}

Vitesse du mélange $: \mu_{\mathrm{m}} \mathrm{V}_{\mathrm{m}}=\mu_{\mathrm{f}} \mathrm{V}_{\mathrm{f}}+\mu_{\mathrm{s}} \mathrm{V}_{\mathrm{s}}$

Vitesse de la phase solide $: v_{s}(y)=D \frac{\left(1-e^{-A h}\right) e^{A y}-\left(1-e^{A h}\right) e^{-A y}}{e^{A h}-e^{-A h}}+B\left(y^{2}-h y\right)-D$

Vitesse de la phase fluide $: v_{f}(y)=C \frac{\left(1-e^{-A h}\right) e^{A y}-\left(1-e^{A h}\right) e^{-A y}}{e^{A h}-e^{-A h}}+B\left(y^{2}-h y\right)-C$

avec $\quad \mathrm{A}=\sqrt{\frac{\mathrm{k}\left(\mu_{\mathrm{s}}+\mu_{\mathrm{f}}\right)}{\mu_{\mathrm{s}} \mu_{\mathrm{f}}}}, \quad \mathrm{B}=\frac{\Delta \mathrm{p}}{2 \mathrm{l}\left(\mu_{\mathrm{f}}+\mu_{\mathrm{s}}\right)}, \quad \mathrm{C}=\frac{\Delta \mathrm{p}}{\mathrm{kl}}\left[\frac{\mu_{\mathrm{s}}^{2} \Phi_{\mathrm{f}}-\left(1-\Phi_{\mathrm{f}}\right) \mu_{\mathrm{f}} \mu_{\mathrm{s}}}{\left(\mu_{\mathrm{f}}+\mu_{\mathrm{s}}\right)^{2}}\right]$

et $\quad \mathrm{D}=\frac{\Delta \mathrm{p}}{\mathrm{kl}}\left[\frac{\mu_{\mathrm{f}}^{2} \Phi_{\mathrm{s}}-\left(1-\Phi_{\mathrm{s}}\right) \mu_{\mathrm{f}} \mu_{\mathrm{s}}}{\left(\mu_{\mathrm{f}}+\mu_{\mathrm{s}}\right)^{2}}\right]$

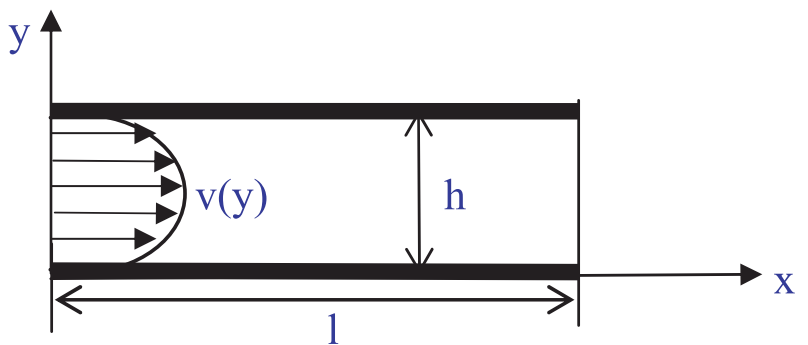

Fig. 9. Écoulement de Poiseuille entre deux plaques parallèles fixes, utilisé pour l'identification de la viscosité de phases solide et fluide du mélange.

Le choix du couple $\left(\mu_{s}, \mu_{f}\right)$ qui vérifie cette condition doit être respecté pour différents diamètres de capillaire. Ces conditions fixent le couple qui doit être utilisé dans la simulation. La solution possible en $\left(\mu_{s}, \mu_{f}\right)$ est représentée figure 10, pour trois diamètres de capillaires. On note qu'il existe deux points d'intersection uniques des faisceaux de courbes correspondant aux différentes épaisseurs de capillaires. Ces deux points représentent deux solutions possibles du problème.

Dans le cas de la figure 10, trois capillaires ont été utilisés. Ils sont caractérisés respectivement par les épaisseurs $h^{(1)}=1,25 \mathrm{~mm}, h^{(2)}=2,5 \mathrm{~mm}, h^{(3)}=5,0 \mathrm{~mm}$. Les variables suivantes ont été utilisées : $\mu_{m}=28,33$ Pa.s ; $\phi_{s}=0,55 ; \dot{\gamma}_{x y}=1179 \mathrm{~s}^{-1} ; L=80 \times 10^{-3} \mathrm{~m}$;

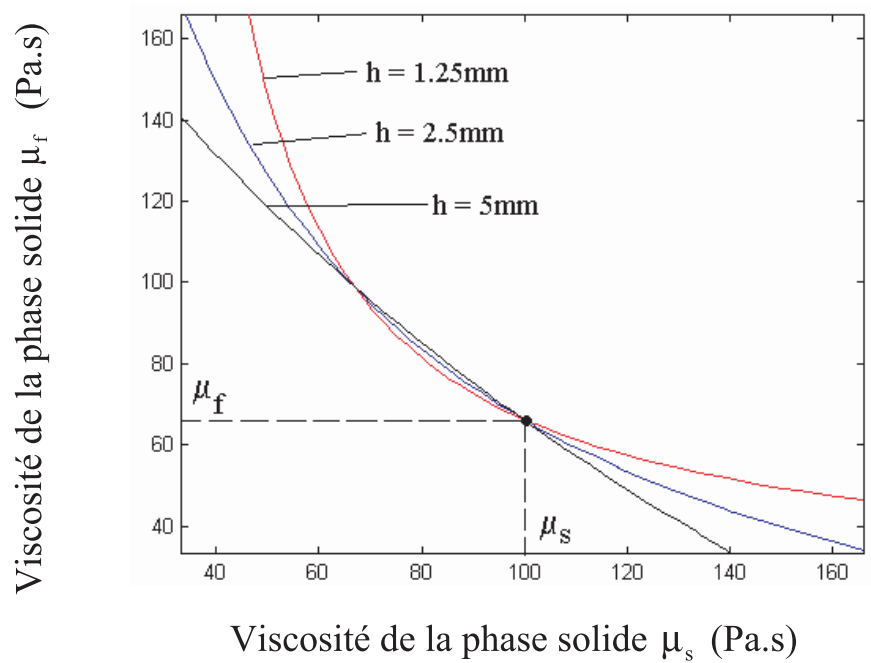

Fig. 10. Résultat de l'identification pour un taux de cisaillement donné pour le mélange polymère fondu + poudres 316L.

$k=1 \times 10^{8}$ Pa.s.m ${ }^{-2}$. La solution est symétrique. La solution correspondant $\mu_{s}>\mu_{f}$ est choisie et est appliquée pour les simulations numériques du moulage par injection métallique.

Les mêmes procédures peuvent être répétées pour différents états rhéologiques et donnent par conséquence 

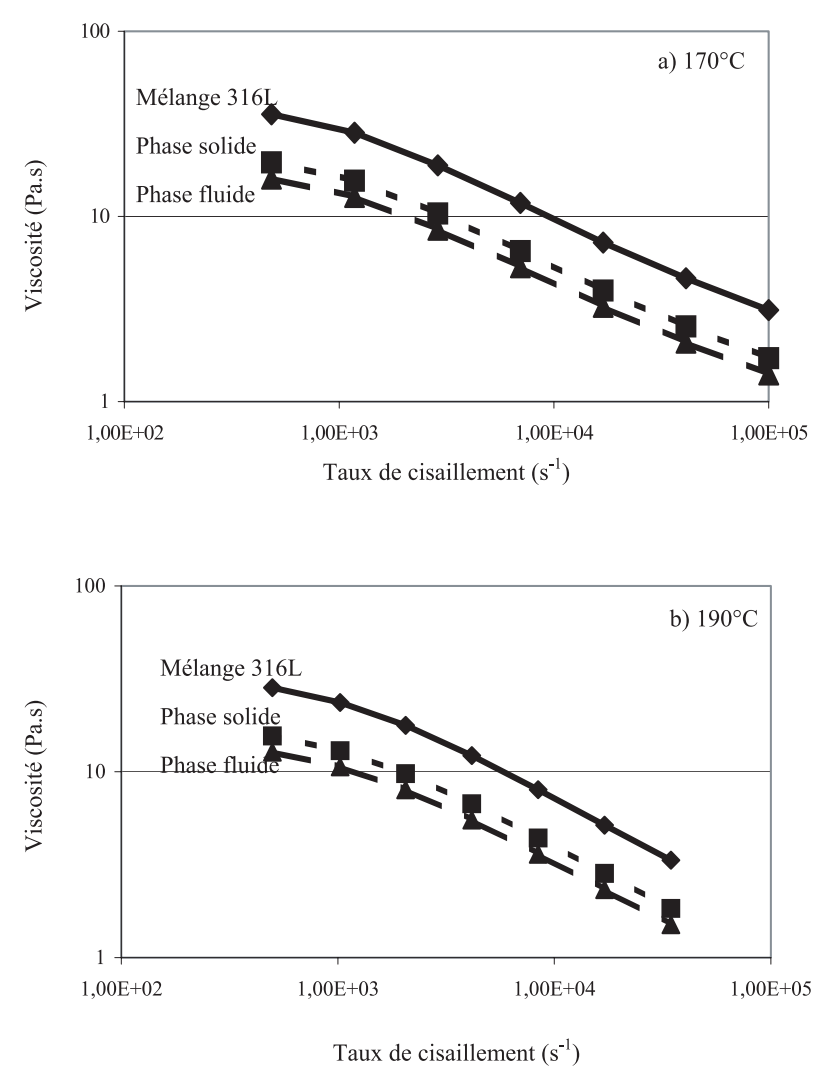

Fig. 11. Lois de viscosité des phases solide et fluide en fonction du taux de cisaillement pour le mélange polymère fondu + poudres $316 \mathrm{~L}$, pour deux températures différentes $\left(T=170{ }^{\circ} \mathrm{C}\right.$ et $\left.T=190{ }^{\circ} \mathrm{C}\right)$.

les lois de viscosité de la phase solide et de la phase fluide en fonction du taux de cisaillement, pour deux températures différentes, figure 11.

\section{Moulage par injection d'une éprouvette de traction}

L'injection des éprouvettes de traction de type 1a-b mentionnées à la section 2 a été utilisée comme cas test afin de réaliser des comparaisons calculs-expériences. Les paramètres d'injection sont donnés sur la figure 12. La pression d'injection est identique à celle utilisée lors des essais avec le moule multi-empreintes. Deux simulations sont réalisées pour l'éprouvette de traction : la première pour la cavité avec un point d'injection, la seconde pour la cavité avec deux points d'injection aux deux extrémités, afin d'étudier la soudure au niveau de la jonction des fronts d'écoulement du mélange.

Le maillage éléments finis utilisé est représenté figure 12. Un évent doit être placé pour évacuer l'air au fur et à mesure du remplissage de l'empreinte afin que celui-ci puisse être complet. Dans le cas étudié, l'évent est placé au milieu de l'empreinte pour l'éprouvette biinjectée et à droite pour l'éprouvette avec un seul point d'injection, afin de rendre compte de la sortie de l'air des empreintes du moule.

\section{Mélange 316L à déliantage thermique}

Maillage :

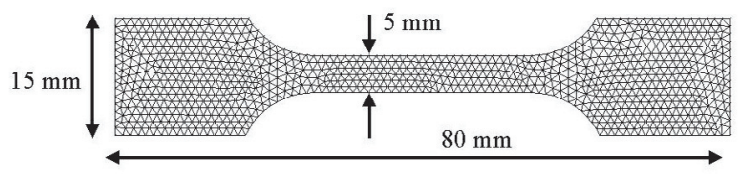

Pression imposée à l'entrée:

$16 \mathrm{MPa}$

Fraction volumique de poudres:

$55 \%$

Terme d'interaction:

Température de la matière

Température du moule

Fig. 12. Paramètres d'injection et maillage utilité pour la simulation de l'injection d'une éprouvette de traction.

Une pression de $16 \mathrm{MPa}$ est imposée au niveau des points d'injection. La simulation numérique révèle un champ de pression qui varie de la valeur maximale imposée à l'entrée à une valeur nulle qui correspond à la sortie ou évent, figure 13a. Dans l'autre cas, le champ de pression varie de la valeur maximale à une valeur nulle, de manière symétrique par rapport au centre de l'éprouvette où se trouve l'évent, figure 13b.

L'état de remplissage correspondant à un taux de remplissage de $80 \%$ est représenté figure 14a. Pour l'empreinte de moule comportant une seule entrée, on observe un remplissage allant du point d'injection vers l'évent, alors que pour deux entrées il est symétrique tout au long du remplissage, figure 14b.

La répartition du champ de température montre une distribution de température symétrique et plus homogène dans le cas d'une éprouvette de type $1 \mathrm{~b}$, figures $15 \mathrm{a}$ et b. La température la plus faible se situe au niveau du contact entre le fluide et la paroi du moule.

À la fin du remplissage, on observe une fraction volumique de poudres de 0,565 pour l'éprouvette avec un point d'injection et de 0,55 pour l'éprouvette deux points d'injection, figure 16. Dans les 2 cas, les fractions volumiques de poudres métalliques sont presque identiques. Il en résulte que si les étapes de déliantage et de densification sont réalisées correctement, les deux éprouvettes ont les mêmes propriétés mécaniques, ce qui a été vérifié par les auteurs grâce à des essais mécaniques [6].

Une comparaison entre les distributions de densités mesurées le long d'une éprouvette de traction injectée avec un point ou deux points d'injection est représentée figure 16 et révèle que la présence de deux points d'injection est favorable. La figure 17 révèle que les évolutions expérimentales et numériques de densités sont similaires, un écart maximal de 4,7\% étant obtenu. Une meilleure 


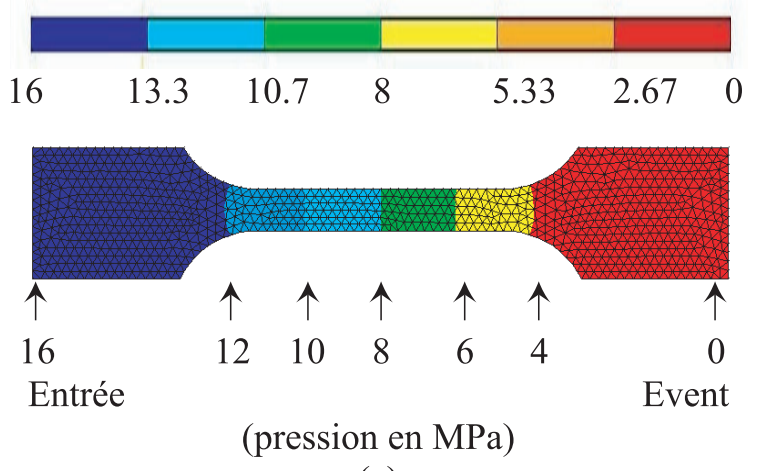

(a)

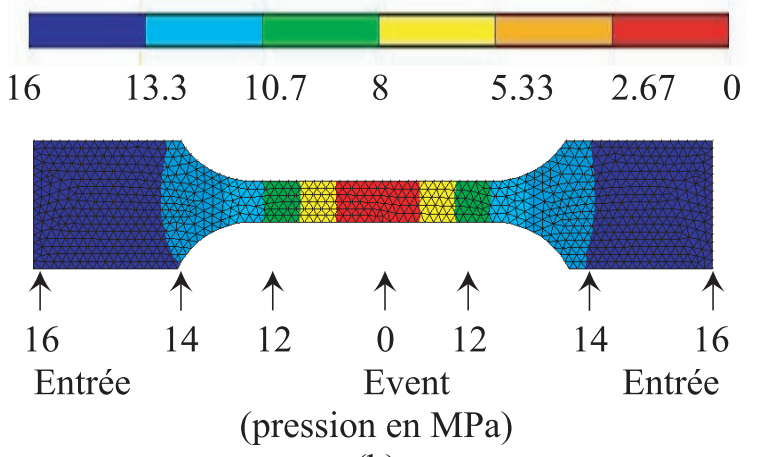

(b)

Fig. 13. Champs de pression, (a) éprouvette injectée avec un point d'injection, (b) éprouvette injectée avec deux points d'injection (pour le mélange polymère fondu + poudre 316L).

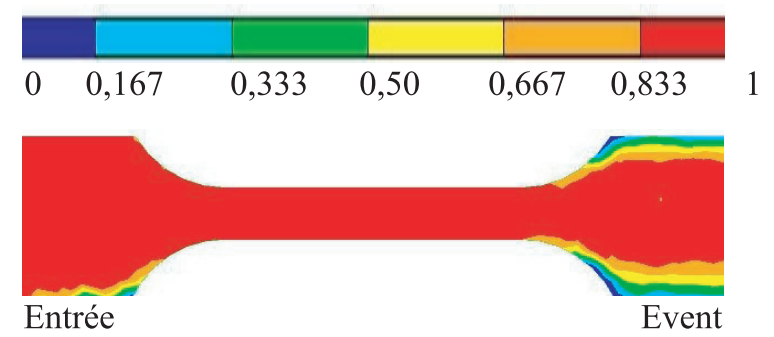

(a) Eprouvette type 1a
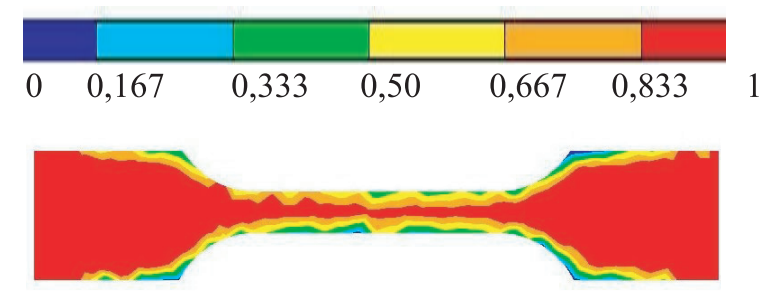

Entrée

Event Entrée

(b) Eprouvette type 1b

Fig. 14. Champ de remplissage $F$ pour un état de remplissage de $80 \%$, (a) éprouvette injectée avec un point d'injection, (b) éprouvette injectée avec deux points d'injection (pour le mélange polymère fondu + poudre $316 \mathrm{~L}$ ).

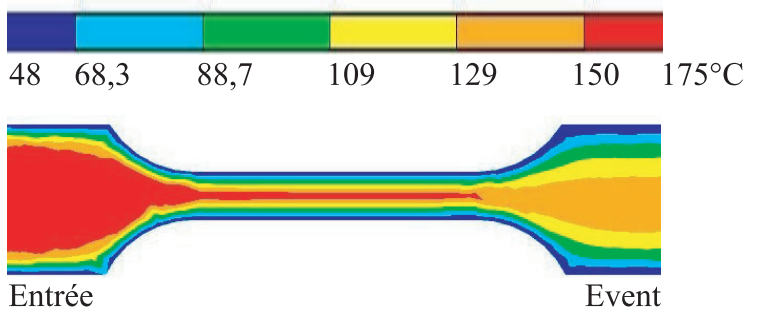

(a) Eprouvette type 1a

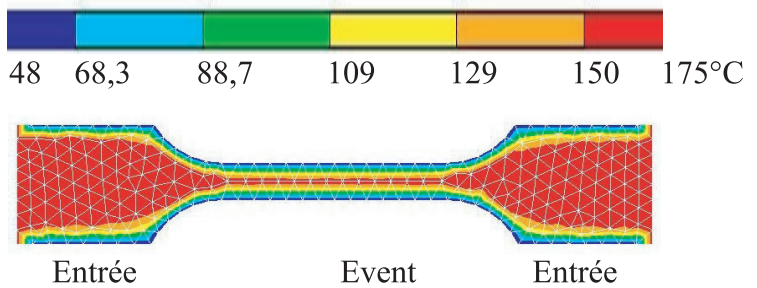

(b) Eprouvette type $1 \mathrm{~b}$

Fig. 15. Champ de température pour un état de remplissage de $80 \%$, (a) éprouvette injectée avec un point d'injection, (b) éprouvette injectée avec deux points d'injection (pour le mélange polymère fondu + poudre $316 \mathrm{~L}$ ).

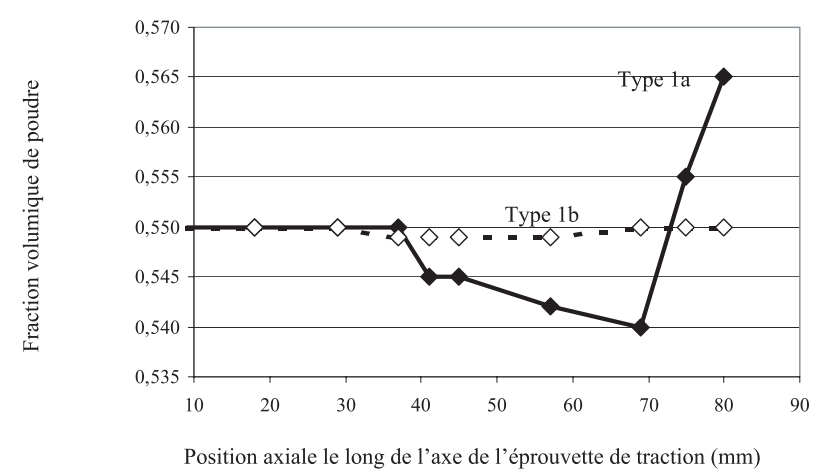

Fig. 16. (b) Fractions volumiques solides moyennes à la fin de remplissage, (a) éprouvette injectée avec un point d'injection, (b) éprouvette injectée avec deux points d'injection (pour le mélange polymère fondu + poudre $316 \mathrm{~L})$.

identification du coefficient d'interaction entre phases permettrait sans doute de réduire cet écart.

\section{Conclusions}

Les écoulements de polymères fondus chargés en poudres métalliques ont été étudiés dans le cas du Moulage par Injection de Poudres Métalliques. Un ensemble expérimental a été développé permettant la réalisation de différents composants, et la répétitivité des résultats a été analysée de façon précise, tant en termes de masse que de dimensions finales. La densité locale a pu être déterminée expérimentalement, ce qui permet d'accéder à la fraction volumique de poudres après injection. Un modèle d'écoulement bi-phasique, basé sur la théorie des mélanges a été élaboré, puis discrétisé par la méthode des 


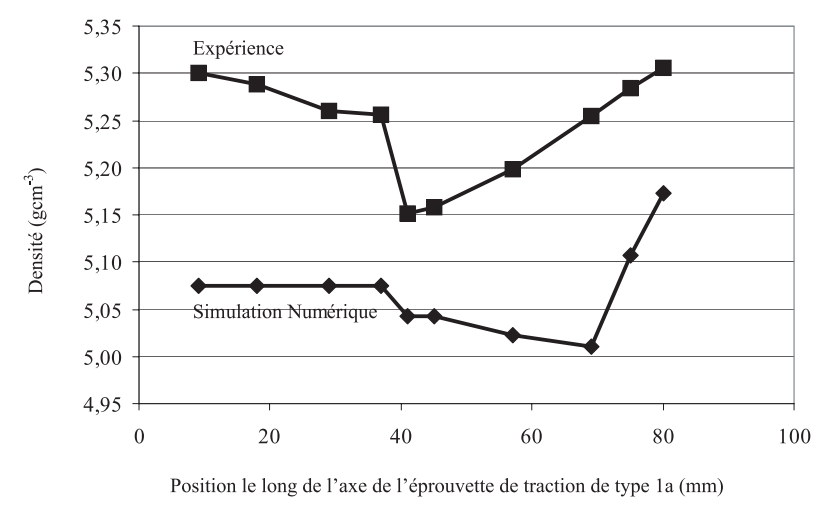

Fig. 17. Comparaison des densitées expérimentales et numériques pour une éprouvette mono-injectée.

éléments finis. Un nouvel algorithme à pas fractionnés a été développé, permettant d'accéder à la variation de fraction volumique des phases avec précision. Des comparaisons entre résultats numériques et expérimentaux, au niveau de la distribution de fraction volumique de poudres métalliques obtenue après injection, prouvent la pertinence de l'approche proposée. Cette approche permet de rendre compte avec précision des problèmes de ségrégation pouvant survenir lors de la phase d'injection en moulage par injection de poudres, y compris pour des cas industriels 3D.

\section{Références}

[1] S. Eroglu, T. Baykara, Effects of powder mixing technique and tungsten powder size on the properties of tungsten heavy alloys, J. Material Processing and Technology 103 (2000) 288-292

[2] T. Shimiza, Y. Murakoshi, K. Wechwitayakhlung, T. Sano, H. Negishi, Characterization of the molding methods and the binder system in the MIM process, J. Material Processing and Technology 63 (1997) 753-758

[3] D.M. Liu, W.J. Tseng, Binder removal from injection moulded zirconia ceramics, Ceramics International 25 (1999) 529-534

[4] R.M. German, A. Bose, Injection of metals and ceramics, Princeton, USA, 1997

[5] R.M. German, Sintering theory and practise, John Wiley \& Sons, New York, 1996

[6] T. Barrière, Expérimentations, Modélisation et Simulation Numérique du Moulage par Injection de Poudres Métalliques, Thèse de doctorat, Université de Franche-Comté, Besançon, 2000

[7] T. Barrière, D. Renault, J.C. Gélin, M. Dutilly, Moulage par injection de poudres métalliques, Expérimentations, modélisation et simulation, Mécanique \& Industries 1 (2000) 201-212

[8] T. Barrière, J.C. Gélin, B. Liu, Experimental and numerical investigations on the properties and quality of parts produced by MIM, Powder Metallurgy 44(3) (2001) 228-234

[9] T. Barrière, J.C. Gélin, B. Liu, Analysis of phase segregation effects arising in fluid-particle flows during metal injection molding, Int. J. Forming Process 4(3-4) (2001) 199-216
[10] T. Barrière, B. Liu, J.C. Gélin, Improving mould design and injection parameters in metals injection moulding by accurate 3D finite element simulation, J. Materials Processing and Technology 125-126 (2002) 518-524

[11] J.C. Gélin, T. Barrière, M. Dutilly, Experiments and computational modeling of metal injection molding for forming small parts, Annals of the CIRP 48(1) (1999) $179-182$

[12] A. Louge, Étude théorique et expérimentale du comportement et de la ségrégation de milieux pâteux lors de l'extrusion, Thèse de doctorat, Université de la Méditerranée, Aix-Marseille, 1996

[13] A. Acrivos, Shear-induced particle diffusion in concentrated suspensions of non colloidal particles, J. Rheol. 39(5) (1995) 813-826

[14] V.V. Bilovol, L. Kowalski, J. Duszczyk, Application of fully $3 \mathrm{D}$ simulation for studying of pressure development during powder injection moulding process, P.I.M 2000, R.M. German (ed.), Pennsylvania State University, USA, 2000, 25-29

[15] K.M. Kulkarni, Factors affecting dimentional precision of MIM Parts under production conditions, Advances Powder Metallurgy and Particulate Materials 19 (1996) $157-170$

[16] J.C. Gélin, T. Barrière, B. Liu, Mould design methods by experiment and numerical simulation in metal injection molding, J. Engineering Manufacture 126, Part B (2002) 1533-1547

[17] Catamold@, Feedstock for powder Injection Molding : Processing-Properties-Application, Technical Information, BASF (1997) 1-10

[18] T. Hartwig, G. Veltl, F. Petzoldt, R. Scholl, B. Kieback, Powders for metal injection molding, J. European Ceramic Society 18 (1998) 1211-1216

[19] T. Iwai, T. Aizawa, J. Kihara, Three dimensional granular modeling for metal injection molding, Powder Metallurgy World Congress, PM'94, ed. S.F.2M. and E.P.M.A, Paris 6-9 juin 2 (1994) 1097-1100

[20] R.M. Bowen, (Ed.) Theory of Mixtures, Continuum Physics, Academic Press, New York, 3, 1976

[21] M. Dutilly, Modélisation du moulage par injection de poudres métalliques, Thèse de doctorat, Université de Franche-Comté, Besançon, 1998

[22] B. Lanteri, H. Burlet, A. Poitou, I. Campion, Powder injection molding, an original simulation of paste flow, Eur. J. Mech., A/Solids 15(3) (1996) 465-485

[23] P.M. Gresho, S.T. Chan, R.L. Lee, C.D. Upson, A modified finite element method for solving the time dependent, incompressible Navier-Stokes equations. Part 1 and Part 2, Int. J. Numer. Methods in Fluids 4 (1984) $557-598$

[24] A.S. Usmani, J.T. Cross, R.W. Lewis, A finite element model for the simulations of mould filling in metal casting and the associated heat transfer, Int. J. Numer. Methods in Engrg. 35 (1992) 787-806

[25] R.W. Lewis, H.C. Huang, A.S. Usmani, J.T. Cross, Finite element analysis of heat transfer and flow problems using adaptive remeshing including application to solidification problems, Int. J. Numer. Methods in Engrg. 32 (1991) $767-781$

[26] R. Lohner, K. Morgan, O.C. Zienkiewicz, The solution of non linear hyperbolic equation systems by finite element method, Int. J. Numer. Methods in Fluids 4 (1984) 1043-1063 